\title{
Estimation of Parameter in a New Truncated Distribution
}

\author{
G. Nanjundan \\ Department of Statistics, Bangalore University, Bangalore, India \\ Email: nanzundan@gmail.com
}

Received June 1, 2013; revised July 5, 2013; accepted July 12, 2013

Copyright (C) 2013 G. Nanjundan. This is an open access article distributed under the Creative Commons Attribution License, which permits unrestricted use, distribution, and reproduction in any medium, provided the original work is properly cited.

\section{ABSTRACT}

This paper discusses the estimation of the parameter in a truncated form of a discrete distribution which is analogous to Burr distribution. The maximum likelihood and the moment estimators of the parameter are obtained. Their asymptotic properties are also established.

Keywords: Maximum Likelihood and Moment Estimators; Fisher Information; Asymptotic Relative Efficiency

\section{Introduction}

Sreehari [1] has derived a class of discrete distributions analogous to Burr family by solving a differential equation. The probability mass function (pmf) of the random variable $X$ having the d-th class of the distributions derived by Sreehari (2010) is

$$
p_{1}(x, \theta)=\left\{\begin{array}{lc}
(x+1-\theta) \cdot \frac{\theta^{x}}{(x+1) !}, & x=0,1,2, \cdots \\
0, & \text { otherwise }
\end{array}\right.
$$

with $0<\theta<1$.

Nanjundan and Naika [2] have discussed the estimation of the parameter of this distribution.

When this distribution is truncated at 0 , the pmf of $X$ turns out to be

$$
p(x, \theta)=\left\{\begin{array}{lc}
(x+1-\theta) \cdot \frac{\theta^{x-1}}{(x+1) !}, x=1,2, \cdots ; 0<\theta<1 \\
0, \quad \text { otherwise. }
\end{array}\right.
$$

We get

$$
E(X)=\frac{1}{\theta}\left(\mathrm{e}^{\theta}-1\right)
$$

and

$$
\operatorname{Var}(X)=2 \mathrm{e}^{\theta}-\frac{1}{\theta}\left(\mathrm{e}^{\theta}-1\right)-\frac{1}{\theta^{2}}\left(\mathrm{e}^{\theta}-1\right)^{2} .
$$

It is straightforward to observe that $E(X)>\operatorname{Var}(X)$. That is this truncated distribution is under dispersed.

The Fisher information measure is computed in Section 2. The maximum likelihood (ML) estimation of $\theta$ is discussed in Section 3 whereas the method of moment estimate of $\theta$ is obtained in Section 4. An asymptotic comparison of the maximum likelihood and method of moment estimates is done in Section 5. The results of a simulation study are presented in Section 6.

\section{Fisher Information Measure}

When $X$ has the pmf specified in (2), we get

$$
\log p(x, \theta)=\log (x+1-\theta)+(x-1) \log \theta-\text { const. }
$$

Then

$$
\frac{\mathrm{d} \log p(x, \theta)}{\mathrm{d} \theta}=-\frac{1}{x+1-\theta}+\frac{x-1}{\theta}
$$

and

$$
\frac{\mathrm{d}^{2} \log p(x, \theta)}{\mathrm{d} \theta^{2}}=-\frac{1}{(x+1-\theta)^{2}}-\frac{x-1}{\theta^{2}} .
$$

The Fisher information measure corresponding to this pmf is given by

$$
\begin{aligned}
I(\theta)= & E\left(-\frac{\mathrm{d}^{2} \log p(x, \theta)}{\mathrm{d} \theta^{2}}\right) \\
= & \sum_{x=1}^{\infty}\left[\frac{1}{(x+1-\theta)^{2}}+\frac{x-1}{\theta^{2}}\right] p(x) \\
= & \sum_{x=1}^{\infty} \frac{1}{x+1-\theta} \frac{\theta^{x-1}}{(x+1) !}+\frac{1}{\theta^{2}} \sum_{x=1}^{\infty} x(x+1-\theta) \cdot \frac{\theta^{x-1}}{(x+1) !} \\
& -\frac{1}{\theta^{2}} \sum_{x=1}^{\infty}(x+1-\theta) \frac{\theta^{x-1}}{(x+1) !} \\
= & \sum_{x=1}^{\infty} \frac{1}{x+1-\theta} \frac{\theta^{x-1}}{(x+1) !}+\frac{1}{\theta^{3}}\left(e^{\theta}-1-\theta\right) .
\end{aligned}
$$


Since the infinite series

$$
\sum_{x=1}^{\infty} \frac{1}{x+1-\theta} \frac{\theta^{x-1}}{(x+1) !}
$$

is not tractable, it can be numerically evaluated for the required values of $\theta$.

Since $0<\theta<1,1-\theta>0$. Hence,

$$
\begin{aligned}
\sum_{x=1}^{\infty} \frac{1}{x+1-\theta} \frac{\theta^{x-1}}{(x+1) !} & \ll \sum_{x=1}^{\infty} \frac{1}{x} \frac{\theta^{x-1}}{(x+1) !} \\
& \ll \sum_{x=1}^{\infty} \frac{\theta^{x-1}}{(x+1) !}=\frac{1}{\theta^{2}}\left(\mathrm{e}^{\theta}-1-\theta\right) .
\end{aligned}
$$

Therefore,

$$
\sum_{x=1}^{\infty} \frac{1}{x+1-\theta} \frac{\theta^{x-1}}{(x+1) !}
$$

is convergent and it can be evaluated numerically.

\section{Maximum Likelihood Estimation}

Let $\underline{X}=\left(X_{1}, X_{2}, \ldots, X_{n}\right)$ be a random sample on $X$ having the pmf specified in (2). Then the likelihood corresponding to the sample is

$$
\begin{aligned}
L(\theta \mid \underline{x}) & =\prod_{j=1}^{n} P\left(X_{j}=x_{j}\right) \\
& =\prod_{j=1}^{n}\left(x_{j}+1-\theta\right) \cdot \frac{\theta^{x_{j}-1}}{(x+1) !}
\end{aligned}
$$

The log-likelihood becomes

$$
\begin{aligned}
& \log L(\theta \mid \underline{x})=\sum_{j=1}^{n} \log \left(x_{j}+1-\theta\right) \\
& +(\log \theta) \sum_{j=1}^{n}\left(x_{j}-1\right)-\text { constant }
\end{aligned}
$$

The maximum likelihood (ML) estimator is given by

$$
\frac{\mathrm{d} \log L(\theta \mid \underline{x})}{\mathrm{d} \theta}=0,
$$

and hence the ML estimator of $\theta$ is the solution of

$$
\sum_{j=1}^{n} \frac{\theta}{x_{j}+1-\theta}-n(\bar{x}-1)=0 .
$$

Since (3) does not yield a closed form expression for the ML estimator, it can be evaluated by a numerical procedure like Newton-Raphson method. Let $\hat{\theta}_{\text {mle }}$ denote the ML estimator of $\theta$.

The pmf in (2) satisfies the following regularity conditions.

1) The support $S=\{x: p(x, \theta)>0\}$ of $X$ does not depend on the parameter $\theta$.

2) The parameter space $(0,1)$ is an open interval.

3) $\log p(x, \theta)$ is twice differentiable w.r.t. $\theta$.
4)

$$
\sum_{x=1}^{\infty} p(x, \theta)=1
$$

is twice differentiable w.r.t. $\theta$ under the summation sign.

5) There exists a function $M(x)$ such that

$$
\left|\frac{\partial^{3} \log p(x, \theta)}{\partial \theta^{3}}\right| \leq M(x)
$$

and

$$
E_{\theta}[M(X)]<\infty .
$$

The conditions 1)-5) are easy to verify. We prove the validity of 5).

Here

$$
\left|\frac{\partial^{3} \log p(x, \theta)}{\partial \theta^{3}}\right|=2\left|\frac{1}{(x+1-\theta)^{3}}+\frac{x-1}{\theta^{3}}\right| \leq 2\left[\frac{1}{x}+\frac{x}{\theta^{3}}\right]
$$

since $0<1-\theta<1$.

$$
\begin{gathered}
\leq 2\left[1+\frac{x}{\left(\theta_{0}-\varepsilon\right)^{3}}\right]=M(x), \\
\forall \theta \in\left(\theta_{0}-\varepsilon, \theta_{0}+\varepsilon\right), \varepsilon>0, x \geq 1,
\end{gathered}
$$

where $\theta_{0}$ is the true value of the parameter. Since the parameter space is an open interval such a neighborhood exists. Obviously, $E_{\theta}[M(X)]<\infty$.

Therefore, $p(x, \theta)$ satisfies all the regularity conditions of Cramer [3] and it belongs to Cramer family.

Hence

$$
\sqrt{n}\left(\hat{\theta}_{m l e}-\theta\right) \stackrel{L}{\longrightarrow} Z \sim N\left(0, \frac{1}{I(\theta)}\right) \text {, as } n \rightarrow \infty .
$$

In other words, $\hat{\theta}_{m l e}$ is consistent and asymptotically normal (CAN) for $\theta$.

\section{Method of Moment Estimation}

Let $\underline{X}=\left(X_{1}, X_{2}, \cdots, X_{n}\right)$ be a random sample on $X$ having the pmf specified in (1.2) and

$$
M_{1}=\frac{1}{n} \sum_{j=1}^{n} X_{j}
$$

be the first moment of the sample. Since

$$
E(X)=\frac{1}{\theta}\left(\mathrm{e}^{\theta}-1\right),
$$

the method of moment (MM) estimator of $\theta$ is given by

$$
\frac{1}{\theta}\left(\mathrm{e}^{\theta}-1\right)=M_{1} .
$$

Evidently the above equation does not admit a closed form expression of the MM estimator of $\theta$ and hence for 
an observed sample, it has to be numerically computed. Let $\hat{\theta}_{m m e}$ denote the moment estimator of $\theta$.

Since $p(x, \theta)$ does not belong to exponential family, the asymptotic normality of $\hat{\theta}_{m m e}$ is not automatic. We establish this property using the delta method.

Note that $X_{1}, X_{2}, \cdots, X_{n}$ are independent and identically distributed (iid) with

$$
E\left(X_{j}\right)=\mu(\theta)=\frac{1}{\theta}\left(\mathrm{e}^{\theta}-1\right)
$$

and

$$
\operatorname{Var}\left(X_{j}\right)=\sigma^{2}(\theta)=2 \mathrm{e}^{\theta}-\frac{1}{\theta}\left(\mathrm{e}^{\theta}-1\right)-\frac{1}{\theta^{2}}\left(\mathrm{e}^{\theta}-1\right)^{2} .
$$

Note that $\mu^{-1}\left(M_{1}\right)$ is the moment estimate of $\theta$. By Levy-Lindeberg central limit theorem,

$$
\sqrt{n}\left(M_{1}-\mu(\theta)\right) \stackrel{L}{\longrightarrow} Z \sim N\left(0, \sigma^{2}(\theta)\right) \text {, as } n \rightarrow \infty \text {. }
$$

Consider the transformation $\phi=\mu(\theta)$. Then

$$
\begin{aligned}
\frac{\mathrm{d} \mu^{-1}(\phi)}{\mathrm{d} \phi} & =\frac{\mathrm{d} \mu^{-1}(\phi)}{\mathrm{d} \theta} \frac{d \theta}{\delta \phi}=\frac{\mathrm{d} \theta}{\mathrm{d} \theta} \frac{1}{\frac{\mathrm{d} \phi}{\mathrm{d} \theta}} \\
& =\frac{1}{\frac{\mathrm{d} \mu(\theta)}{d \theta}}=\left(\frac{\mathrm{d} \mu(\theta)}{\mathrm{d} \theta}\right)^{-1}
\end{aligned}
$$

Also

$$
\sigma^{2}\left(\mu^{-1}(\phi)\right)=\sigma^{2}(\theta)
$$

Here

$$
\frac{\mathrm{d} \mu}{\mathrm{d} \theta}=\frac{\theta \mathrm{e}^{\theta}-\mathrm{e}^{\theta}+1}{\theta^{2}}, 0<\theta<1 .
$$

Therefore,

$$
\left[\frac{\mathrm{d} \mu}{\mathrm{d} \theta}\right]^{-1}=\frac{\theta^{2}}{\theta \mathrm{e}^{\theta}-\mathrm{e}^{\theta}+1} \neq 0
$$

and is continuous.

Hence by the delta method (see Knight [4]),

$$
\sqrt{n}\left(\mu^{-1}\left(M_{1}\right)-\theta\right) \stackrel{L}{\longrightarrow} Z \sim N\left(0, \sigma^{2}(\theta)\left[\frac{\mathrm{d} \mu}{\mathrm{d} \theta}\right]^{-2}\right),
$$

as $n \rightarrow \infty$,

that is $\hat{\theta}_{m m e}$ is also CAN for $\theta$.

\section{Asymptotic Relative Efficiency}

The asymptotic relative efficiency of the ML estimator over the method of moment estimator is given by

$$
A R E=\frac{\text { Asymptotic variance of } \hat{\theta}_{m m e}}{\text { Asymptotic variance of } \hat{\theta}_{m l e}} \text {. }
$$

Since the asymptotic variance of $\hat{\theta}_{m l e}$ has no closed form expression and is computed numerically, the ARE has also to be numerically evaluated. The Table 1 shows the ARE for various values of $\theta$.

The ARE of the ML estimator over the MM estimator is steadily increasing as $\theta$ increases. That is the ML estimator uniformly performs better than the MM estimator.

\section{Simulation Study}

A modest simulation study has been carried out using the R software. One thousand samples, of size 100, 250, and 500 were generated from the truncated distribution specified in (2) for $\theta=0.5$. Both the ML and the MM estimates were computed solving respectively (3) and (4) using Newton-Raphson method. Sample means were taken to be the initial estimates. Since the asymptotic normality of both estimates has been analytically established, an elaborate simulation study has not been done. However the following histograms give graphical evidence of the asymptotic normality of the estimates. As the sample size increases, the estimates tend to be distributed more normally.

The Figures 1-3 show the histograms of the MLEs and the MMEs based on 1000 samples of sizes 100, 250, and 500 and $\theta=0.5$.

Table 1. The ARE of the MLE over the MME.

\begin{tabular}{ccccc}
\hline & 0.1 & 0.2 & 0.3 & 0.4 \\
\hline ARE & 1.100273 & 1.189627 & 1.268874 & 1.338841 \\
& 0.5 & 0.6 & 0.7 & 0.8 \\
ARE & 1.400382 & 1.454398 & 1.501861 & 1.543859 \\
\hline
\end{tabular}

Histogram of mle

Histogram of mme
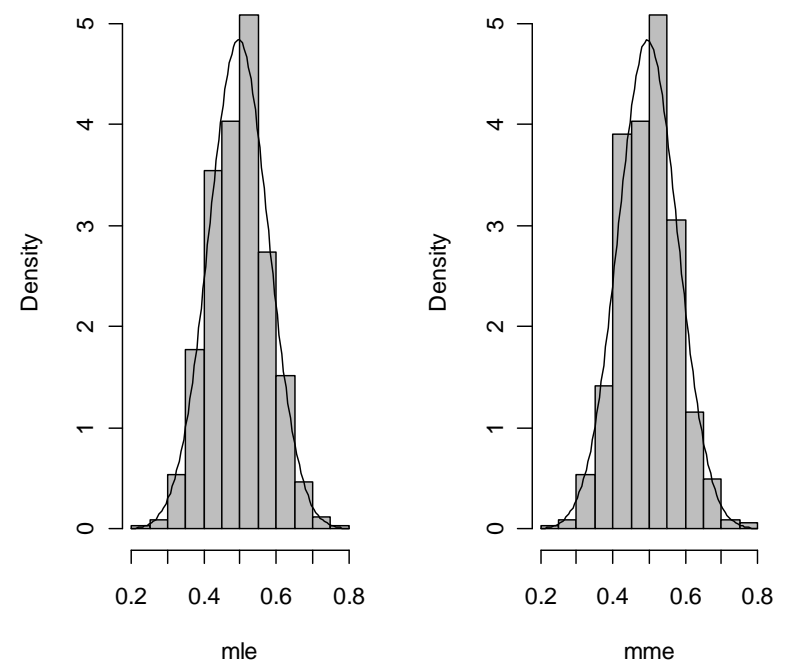

Figure 1. The histograms of the MLEs and the MMEs for sample size 100 . 
Histogram of mle

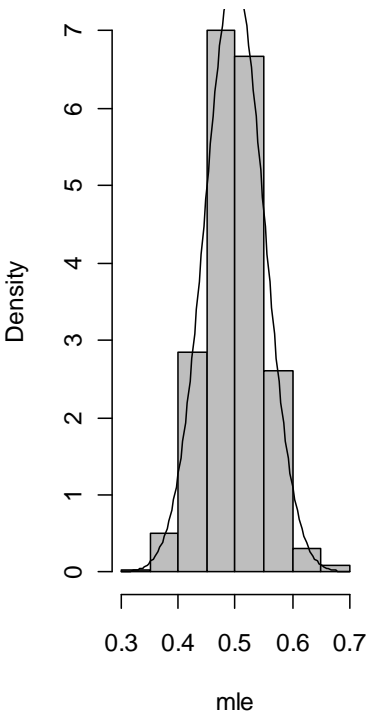

Histogram of mme

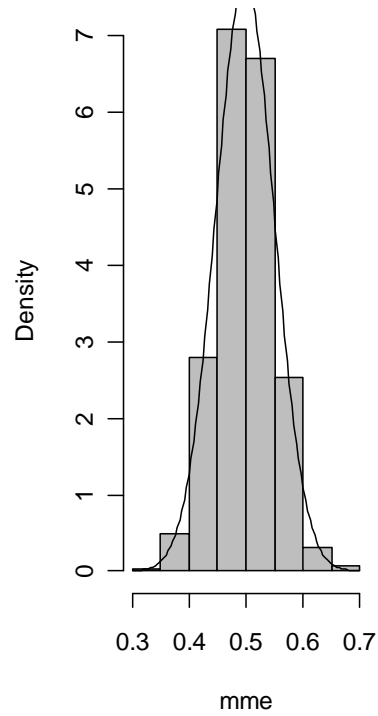

Figure 2. The histograms of the MLEs and the MMEs for sample size 250 .
Histogram of mle

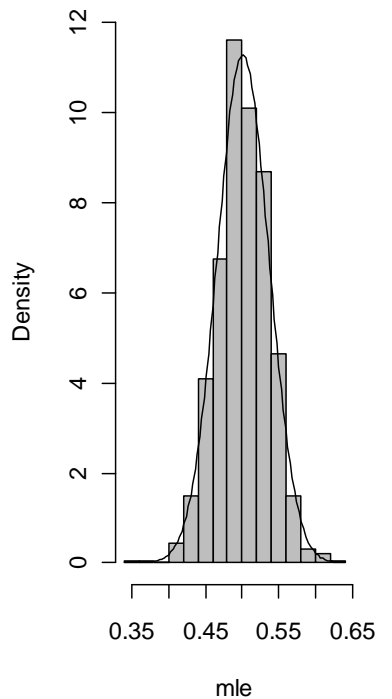

Histogram of mme

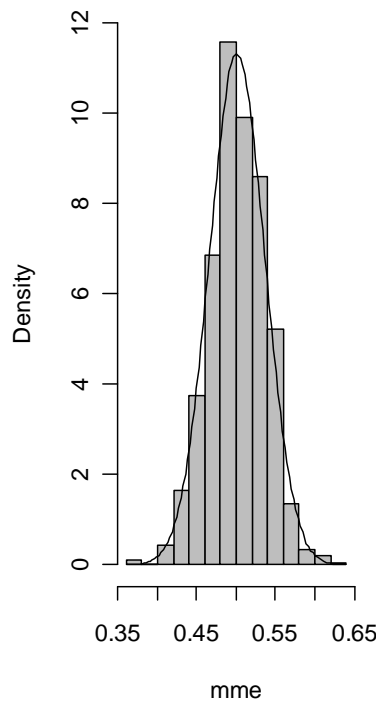

Figure 3. The histograms of the MLEs and the MMEs for sample size 500 .

\section{Discussions and Summary}

The distribution discussed in this paper is structurally similar to the truncated Poisson distribution and under dispersed. Hence this distribution can be used as an alternative to the truncated Poisson when the data exhibit under dispersion.

Both the ML and MM estimators do not have closed form expressions. But they can easily be computed using Newton-Raphson method. Both of the estimators are CAN for the parameter. The ML estimator is asymptotically more efficient than the MM estimator.

\section{Acknowledgements}

The author is grateful to the referee and Prof. B. Chandrasekar whose suggestions improved the presentation of the results.

\section{REFERENCES}

[1] M. Sreehari, "On a Class of Discrete Distributions Analogous to Burr Family,” Journal of Indian Statistical Association, Vol. 46, No. 2, 2008, pp. 163-181.

[2] G. Nanjundan and T. Reevindra Naika, "Estimation of Parameter in a New Discrete Distribution Analogous to Burr Distribution," Proceedings of the International Conference on Information Technology and Computer Application Engineering, 27-28 August 2013, Hong Kong. (To appear).

[3] H. Cramer, "Mathematical Methods of Statistics," Princeton, 11th Print, Princeton University Press, Princeton, 1966.

[4] K. Knight, "Mathematical Statistics,” Chapman and Hall, London, 2000. 